\title{
Two-dimensional Electronic Spectroscopy of Chlorophyll a: Solvent Dependent Spectral Evolution
}

Roberta Moca, S. R. Meech and I. A. Heisler*

School of Chemistry, University of East Anglia, Norwich Research Park, Norwich NR4 7TJ, UK

\section{Abstract}

The interaction of the monomeric chlorophyll Q band electronic transition with solvents of differing physical-chemical properties is investigated through two-dimensional electronic spectroscopy (2DES). Chlorophyll constitutes the key chromophore molecule in light harvesting complexes. It is well known that the surrounding protein in the light harvesting complex fine tunes chlorophyll electronic transitions to optimize energy transfer. Therefore an understanding of the influence of the environment on the monomeric chlorophyll electronic transitions is important. The $Q$ band 2DES are inhomogeneous at early times, particularly in hydrogen bonding polar solvents, but also in nonpolar solvents like cyclohexane. Interestingly this inhomogeneity persists for long times, even up to the nanosecond timescale in some solvents. The reshaping of the 2DES occurs over multiple timescales and was assigned mainly to spectral diffusion. At early times the reshaping is Gaussianlike, hinting at a strong solvent reorganization effect. The temporal evolution of the 2DES response was analysed in terms of a Brownian oscillator model. The spectral densities underpinning the Brownian oscillator fitting were recovered for the different solvents. The absorption spectra and Stokes shift were also properly described by this model. The extent and nature of inhomogeneous broadening was a strong function of solvent, being larger in $\mathrm{H}$-bonding and viscous media and smaller in nonpolar solvents. The fastest spectral reshaping components were assigned to solvent dynamics, modified by interactions with the solute.

Author for correspondencei.heisler@uea.ac.uk 


\section{INTRODUCTION}

The spectroscopy and dynamics associated with the electronic transitions of chlorophyll molecules in light harvesting complexes and reaction centres is an active area of research. ${ }^{1-3}$ The ultimate objective is to unravel the complex interactions underlying the robust and efficient conversion of absorbed solar radiation into chemical energy, with a view to developing more efficient biomimetic energy transduction devices. A key role in the spectroscopy of light harvesting complexes is played by excitonic coupling between chlorophyll molecules precisely oriented and positioned by the surrounding protein matrix. The complex couplings and energy transfer pathways which exist in these complexes are beginning to be resolved through the application of two-dimensional electronic spectroscopy (2DES), which is uniquely sensitive to intermolecular coupling and energy transfer between electronic states. Much interest has been generated by the observation of long lived coherences in the 2DES of light harvesting complexes, manifested as amplitude and phase oscillations, leading to the suggestion that such coherences may play a role in optimising energy transport, or at least act as an important probe of it. One of the difficulties in assigning the origin of oscillations in excitonically coupled chromophores has been in resolving them from oscillations due to intramolecular vibrational modes which are coupled to the molecular electronic transitions. A number of tools have been proposed to achieve this, including the distinct behaviour of the 2DES response either resolved into its rephasing and non-rephasing pathways or observed under different polarization conditions. ${ }^{4-5}$ This remains an active area of research, but it is undoubtedly true that, wherever possible, a study of the chromophore isolated in solution is a great aid in assigning intramolecular vibrational modes in 2DES; such measurements are reported below for chlorophyll a (Chl a).

Intermolecular excitonic coupling may also give rise to marked red or blue shifts in the electronic transition, which can be useful in inferring the existence of couplings and the geometry of the coupled chromophores. ${ }^{6-7}$ However, it is also established for chlorophyll molecules that the environment itself can also give rise to shifts in the electronic spectrum, even for monomeric species. ${ }^{8}$ Different effects are observed for $\mathrm{H}$-bonding and non-H-bonding solvents, solvents of different aromaticity and refractive index and, to a lesser extent polarity. These have been neatly summarized in a recent review. ${ }^{8} \mathrm{~A}$ combination of these factors gives rise to quite marked shifts in the electronic spectra of chlorophylls when they are incorporated into a protein matrix. ${ }^{3}$ How these protein-pigment interactions might modulate 2DES is not immediately clear, thus it is important to characterize the effect of the medium on the 2DES of chlorophylls, which is the main objective of the work described here. 
A number of previous articles have probed the ultrafast dynamics $\mathrm{Chl}$ a in solution. Early work by Sundstrom and co-workers revealed spectral hole burning in the $\mathrm{Q}_{\mathrm{y}}$ transition of $\mathrm{Chl}$ a in pyridine solution, indicating inhomogeneous broadening of this electronic transition. ${ }^{9}$ In addition, picosecond kinetics were observed in pyridine and methanol solutions which occurred in addition to excited state population decay. ${ }^{9-10}$ These sub-picosecond and picosecond dynamics were assigned to spectral diffusion and solvation dynamics, reflecting the interaction of solute and solvent. More recently Kobayashi and co-workers used extremely broadband ultrafast pulses in pump-probe experiments to observe wavepacket dynamics in the $\mathrm{Q}_{\mathrm{y}}$ band of $\mathrm{Chl}$ a in ether:propanol solution. ${ }^{11}$ They observed a number of vibrational modes coupled to the $Q_{y}$ electronic transition and involved in the modulation of the electronic absorption spectrum. Very recently, Wells et al. reported the first 2DES of monomeric $\mathrm{Chl}$ a in methanol. ${ }^{12}$ These authors adapted the centre line slope method from 2D IR spectroscopy, based on a Kubo line-shape analysis, to characterise spectral diffusion dynamics on the tens of picoseconds timescale.

This work presents a significant extension of these previous works by: i) studying $\mathrm{Chl}$ a in a range of solvents and applying the physically more meaningful multimode Brownian oscillator analysis, which correctly accounts for both lineshape broadening as well as spectral Stokes shift; ii) exploiting the high time resolution of $2 D E S$ to assess the early spectral time evolution; iii) the vibrational coherences in $\mathrm{Chl}$ a are analysed through rephasing Fourier Transform amplitude maps. We find a significant solvent dependence in the extent of inhomogeneous broadening and in the dynamics of spectral diffusion which is assigned to specific solute solvent interactions. We also characterise the contribution of solvent modes to observed oscillations in 2DES.

\section{EXPERIMENTAL}

The spectrometer for 2DES measurements has been described in detail elsewhere..$^{13} \mathrm{It}$ is based on a $10 \mathrm{kHz}$ amplified Ti:sapphire laser pumping a NOPA which for these experiments was tuned to give sub $15 \mathrm{fs}$ pulses centred at $660 \mathrm{~nm}$ with a bandwidth of $60 \mathrm{~nm}$. The 2DES spectrometer was designed in the four beam boxcar geometry with the phase-stable beams generated by two beam splitters, and interpulse delays controlled by two precision delay stages, one mounted on a $0.1 \mu \mathrm{m}$ resolution mechanical delay stage. The beams are modulated by a pair of synchronised choppers. The arrangement of the chopper and delay sequences for fast acquisition of the 2DES signal while minimising scattered light and pump-probe contributions, and simultaneously recording the local oscillator and pump-probe spectra, are described in detail elsewhere. ${ }^{13}$ 
The samples were prepared by dissolving $\mathrm{Chl}$ a (Alpha Laboratories) in a variety of spectroscopic grade solvents. $\mathrm{Chl}$ a in solution is known to exhibit both self-quenching and the formation of complexes and aggregates, all of which may perturb the electronic spectra. ${ }^{14}$ To check for such effects measurements were made in $0.2 \mathrm{~mm}$ and $1 \mathrm{~mm}$ cells both with an OD of 0.3 at the maximum of the $S_{0} \rightarrow S_{1}$ transition, thus varying the concentration by a factor of 5 . The main features of the molecular response were independent of concentration confirming that we are studying monomeric Chl a.

\section{RESULTS AND DISCUSSION}

\section{a) 2DES Oscillatory Dynamics}

Figure 1a presents the 2DES spectrum of $\mathrm{Chl}$ a in methanol at a population time of $T=100 \mathrm{fs}$. Two striking features are the elongation along the diagonal, which points to a distribution of electronic transitions (inhomogeneity) and a cross peak below the diagonal. These features will be discussed in the next section but here another feature, not captured by a single spectrum, will be discussed. By stacking together a series of 2D spectra obtained for different population times (for the definition of delay times, see the scheme in Supporting Information, Figure S1), it is possible to observe oscillations for a given pair of excitation-detection frequencies as exemplified in Figure $1 \mathrm{~b}$. In general, electronic transitions are coupled to one or more vibrational transitions and $\mathrm{Chl}$ a is no exception. ${ }^{11}$ For a point $\left(\bar{v}_{1}, \bar{v}_{3}\right)=(15770,14500)$ below the diagonal, an oscillation can clearly be resolved (Figure 1b) whose power spectrum (Figure 1c) produces a peak at $743 \mathrm{~cm}^{-1}$. Although solvent Raman transitions can also contribute signals to 2DES spectra (as discussed in next paragraph), methanol has no significant Raman active modes below $1000 \mathrm{~cm}^{-1}$, therefore the oscillations measured can be assigned to dissolved $\mathrm{Chl}$ a molecules. Further, by analysing different excitation-detection frequency pairs, other vibronic transitions at 255 and $345 \mathrm{~cm}^{-1}$ are also detected. These compare well with the frequencies reported by Du et al. ${ }^{11}$ In order to determine the oscillation amplitude distribution over the whole excitation-detection map, Fourier transform analysis is performed for each pair of excitation-detection frequencies. ${ }^{15}$ Power spectra amplitude maps for the rephasing signal for the frequencies 255, 345 and $745 \mathrm{~cm}^{-1}$ are presented in Supporting Information, Figure S2. As discussed recently, even though Feynman diagrams usefully point at specific regions in the 2D spectra where oscillations are to be expected, the oscillation amplitude distribution is a non-trivial function of excitation-detection frequencies. ${ }^{15}$ 
As mentioned above, care has to be taken when working with solvents that have vibrational modes in the same region as the dissolved solute. For example, when cyclohexane was the solvent, we observed oscillations at ca $800 \mathrm{~cm}^{-1}$. Although this is close to one of the frequencies reported for $\mathrm{Chl}$ a $\left(743 \mathrm{~cm}^{-1}\right)$ it was detected across the 2D map, as shown in Figures S3a an S3b, which present the $T$ dependent oscillating signal for points $A, B$ and $C$ across the 2D spectrum. Even though there is hardly any absorption for the excitation-frequency pair at point $A$, an oscillating signal can still be resolved. At point $B$, where the sample absorbs, intramolecular modes at 255 and $345 \mathrm{~cm}^{-1}$ are also measured, as shown in the power spectrum, Figure S3c (dashed lines). However, the contribution for the mode at $800 \mathrm{~cm}^{-1}$ was greatly reduced by working with higher solute concentrations consistent with an assignment to a Raman active solvent mode. This attribution was further confirmed through the observation that the frequency recovered was dependent on the solvent and that the same measurements in cyclohexane-h12 and cyclohexane-d12 recovered a shifted frequency consistent with the known isotope shift in the Raman spectrum (Supporting Information, Figure S4).

That such a solvent contribution is expected in measurements of a spectrally dispersed transient third order nonlinear optical response was reported previously by Ziegler and co-workers. ${ }^{16}$ As they have shown, there is no net energy exchange between the applied field and the sample. As a result the solvent signal is expected to disappear when the pump-probe response is integrated over all detection frequencies. We have confirmed this prediction with a dilute porphyrin sample, which has the advantage of having its $Q$ band electronic transition coupled to (mainly) a single low frequency vibration. ${ }^{15}$ In this case, we compared the dilute porphyrin sample in toluene with the pure solvent response and recorded the integrated pump-probe response for both (detailed in Supporting Information, Figure S5). The effect of the integration is to remove the solvent Raman mode contributions. Thus, these observations show that, although solvent vibrational modes can make a significant contribution to the oscillatory response observed in 2DES, a number of means exist to distinguish them from the solute response, which is usually the one of most interest.

\section{b) 2DES Reshaping}

The 2D spectral evolution of $\mathrm{Chl}$ a in acetone is presented in Figure 2. As has been described in detail elsewhere, these data result from measurements of the heterodyne detected third order nonlinear optical response function, measured as a function of two time delays, the coherence time ( $\tau$ ) between pulses 1 and 2 and the population ( $T$ ) time between pulses 2 and 3 (Figure S1). ${ }^{17-18}$ The $\bar{v}_{3}$ axis is obtained directly in the frequency domain by spectral interferometry and the $\bar{v}_{1}$ axis by a Fourier transform of the time domain data as a function of the coherence time. The resulting 2DES plot is then measured for a series of population times, as shown in Figure 2. The data are dominated 
by the response associated with the $\mathrm{Q}_{\mathrm{y}}\left(\mathrm{S}_{0}(\mathrm{~V}=0) \rightarrow \mathrm{S}_{1}\left(\mathrm{~V}^{\prime}=0\right)\right)$ electronic transition at $15100 \mathrm{~cm}^{-1}$, and are elongated along the diagonal at early times. However, for increasing $T$ the plot becomes more circular; such an evolution is characteristic of an initially inhomogeneously broadened frequency distribution which relaxes through a variety of mechanisms such as, for example, spectral diffusion. ${ }^{19-21}$ A similar result was reported by Wells et al. for the 2DES of $\mathrm{Chl}$ a in methanol and related observations were reported from hole-burning measurements by Sundstrom and coworkers. ${ }^{9,12}$ The solvent dependence of spectral diffusion in $\mathrm{Chl}$ a will be investigated further below.

In addition to the dominant response associated with the $\mathrm{Q}_{\mathrm{y}}$ electronic transition a shoulder is observed in the $2 \mathrm{D}$ response at $\left(\bar{v}_{1}, \bar{v}_{3}\right) \approx(15000,14000)$. This component, which appears within 50 $\mathrm{fs}$, correlates with the frequency of the first vibronic transition at $\bar{v} \approx 14000 \mathrm{~cm}^{-1}$ in the emission spectrum (green curve in Figure 1a). This spectral region is covered by significant laser spectral intensity (blue curve, Figure 1a). Thus we assign this response to a stimulated emission from the $Q_{y}$ $\left(S_{1}\left(v^{\prime}=0\right) \rightarrow S_{0}(v=1)\right)$ vibronic transition.

Figure 2 shows that as the population time increases the pure electronic response evolves from elliptical to more circular. In the limit of pure static inhomogeneous broadening the response would be spread along the diagonal, while in the opposite limit of a purely homogeneous lineshape a circular 2D plot would be recovered, and in both cases the response would be time independent. ${ }^{21}$ Aggregated systems can display intermolecular electronic energy transfer, which can explain such a spectral reshaping. ${ }^{3}$ However, this can be ruled out by the fact that $\mathrm{Chl} \mathrm{a}$, in nonaqueous solvents at concentrations below $100 \mu \mathrm{M}$, as used in this work, is present as a monomer. The observed temporal evolution from elliptically elongated towards circular in the present case thus indicates spectral diffusion, where fluctuations in the environment surrounding the solute give rise to changes in its electronic transition frequency. Thus, the temporal evolution reflected in the shape of the 2DES peak reveals the correlation function of the electronic transition frequency, which, in turn, correlates with fluctuations in the $\mathrm{Chl}$ a environment. Solvent dynamics are also manifested through the Stokes shift, clearly present in the 2D spectra shown in Figure 2; the central maximum shifts towards lower detection frequencies as $T$ increases, due to a red shift in the stimulated emission. Such spectral diffusion has been analysed to recover the frequency-frequency correlation function (FFCF) for vibrational transitions in 2DIR measurements and for electronic transitions by 2DES and by photo-echo peak-shift measurements. ${ }^{12,} 22-23$

To characterize spectral diffusion in $\mathrm{Chl}$ a in more detail we have measured 2D spectra as a function of $T$ with a high density of data points out to several hundred picoseconds. As discussed before, there are different ways of quantifying the spectral evolution of $2 \mathrm{D}$ signals. ${ }^{19}$ For example, the 
centre-line-slope (CLS), given by a linear fit to the maxima of the response at each detection frequency, has been favoured mainly by 2D IR spectroscopy groups. ${ }^{22}$ Kwak et al showed that, after some assumptions and approximations, the CLS is directly proportional to the FFCF which is the fundamental physical quantity describing spectral diffusion, and which can be obtained by molecular dynamics simulations. ${ }^{22}$ However, the assumptions employed in deriving the CLS might not work so well for 2DES and therefore we report another quantity, proposed by Lazonder et al, which is the ellipticity of the 2D spectra. ${ }^{24}$ Lazonder and Tokmakoff showed that this quantity is identical to the FFCF. ${ }^{19,24}$ The ellipticity can be determined by measuring the 2D spectrum diagonal and antidiagonal full width at half maxima ( $D$ and $A$, respectively) and then calculating the ratio $\left(D^{2}-A^{2}\right) /\left(D^{2}+A^{2}\right)$. The resulting ellipticity, determined for our measured data, is plotted for four representative solvents in Figure 3, grouped according to contrasting polarity (acetone and cyclohexane), H-bonding (acetone and methanol) and viscosity (acetone and ethylene glycol). Immediately apparent is a strong time dependence of the ellipticity and, therefore, the FFCF on the physical-chemical properties of the solvent. Qualitatively, the spectral diffusion becomes much slower in the viscous polar H-bonding solvent ethylene glycol (EG), compared to the fluid solvents methanol (MeOH) and acetone (AC). In the non-polar solvent cyclohexane $(\mathrm{Cyh})$ the initial response is more circular than for all the other solvents, i.e. the initial distribution of transition frequencies is more homogeneous. In addition, the temporal evolution towards the final circular response is much faster and more complete than for any of the polar solvents. Among the polar solvents studied neither the presence of $\mathrm{H}$-bonding nor the value of the dielectric constant has an obvious effect on the correlation function. Thus, these qualitative considerations already reveal a significant dependence of the rate of spectral diffusion in $\mathrm{Chl}$ a on the medium.

To put this analysis on a more quantitative footing we use a phenomenological model of the 2DES response utilising Brownian oscillators (BOs) for the spectral density. The signal field in the third order experiment arises from a convolution of eight third order material response functions with the electric fields of the three excitation pulses. ${ }^{15,18,25}$ Modelling of the experimental data that result requires calculation of these response functions, which can in-turn be obtained from the lineshape function:

$g(t)=\frac{1}{2 \pi} \int_{-\infty}^{\infty} d \omega(1-\cos (\omega t)) \operatorname{coth}\left(\frac{\beta \hbar \omega}{2}\right) \frac{C(\omega)}{\omega^{2}}+\frac{i}{2 \pi} \int_{-\infty}^{\infty} d \omega(\sin (\omega t)-\omega t) \frac{C(\omega)}{\omega^{2}}$,

in which $C(\omega)$ is the spectral density, here represented by a sum of overdamped $\mathrm{BOs}, \mathrm{C}_{O D}(\omega)$ :

$C_{O D}(\omega)=\sum_{i=1}^{3} 2 \lambda_{i} \frac{\omega \Lambda_{i}}{\omega^{2}+\Lambda_{i}^{2}}$ 
where $\lambda$ is a coupling strength and $\Lambda$ the inverse correlation time, and/or by a Gaussian correlation function

$C_{g}(\omega)=\frac{2 \lambda \omega}{\sqrt{2 \pi} \omega_{g}} \exp \left(-\frac{\omega^{2}}{2 \omega_{g}^{2}}\right)$

where $\omega_{g}$ is the Gaussian spectral density central frequency. Utilising (1) - (3) the four response functions required to fit the signal from an electronic two level system (i.e. ignoring the much smaller contribution for the vibronic response which would require additional underdampled BOs) are readily calculated. Finally using the $\lambda, \Lambda$ and $\omega_{g}$ as fitting parameters we simultaneously fit the linear absorption spectrum:

$\sigma_{A B S}=\left|\mu_{e g}\right|^{2} \operatorname{Re}\left[\int_{-\infty}^{\infty} d t \exp \left(i \omega_{e g} t-g(t)\right)\right]$,

where $\omega_{e g}$ is the $S_{0} \rightarrow S_{1}$ transition frequency, and the 2DES are obtained through the expression:

$S^{(3)}\left(\omega_{1}, t_{2}, \omega_{3}\right)=\int_{-\infty}^{\infty} d t_{1} \int_{-\infty}^{\infty} d t_{3} R^{(3)}\left(t_{1}, t_{2}, t_{3}\right) \exp \left(i \omega_{1} t_{1}\right) \exp \left(i \omega_{3} t_{3}\right)$,

where $R^{(3)}\left(t_{1}, t_{2}, t_{3}\right)$ is the sum of the four calculated response functions contributing to the signal for an electronic two-level system.

The calculated 2DES corresponding to the data in Figure 2 are shown in Figure 4a, and the agreement for the dominant electronic response is good. However, a better representation of the evolution is obtained from simultaneously fitting the linear absorption as well as the ellipticity and Stokes shift as a function of the population time. Examples are shown for $\mathrm{Chl}$ a in acetone in Figure $4 \mathrm{~b}$. Fits were obtained with a sum of four overdamped BOs (Equation 2), which was the minimum required for a good fit; fitting parameters as well as graphs for all the experimental and calculated data are presented in Supporting Information, Table S1a and Figures S7-S9, respectively. We did not include an underdamped BO component in order to account for the main vibrational overtone at $16228 \mathrm{~cm}^{-1}$ and therefore the linear absorption is only fit well up to $15530 \mathrm{~cm}^{-1}$. We found that there was no significant contribution to the spectral reshaping by including this term probably because the laser spectrum did not cover this absorption peak. A closer inspection of the initial evolution of the ellipticity (Figure 4b) clearly shows a Gaussian-like relaxation. Therefore, replacing the most highly damped BO with a Gaussian function (Equation 3) resulted in an improved fit at early times (Table S1b). The Stokes shift is also well captured by this analysis, as shown in Figure 4b. Figure 5 presents the recovered spectral densities, corresponding to equations 2 and 3, plotted for each of the five solvents studied. 
The most striking result from this analysis is the extent of residual inhomogeneous broadening even after several hundred picoseconds of relaxation in all polar solvents. This is represented by the lowest frequency response in Figure 5 (peak centred at $0.004 \mathrm{~cm}^{-1}$, corresponding to a frequency of $0.12 \mathrm{GHz}$ ), where a relaxation time longer than the accessible time range in our experiment (i.e. $>1$ nanosecond) was assumed (Tables S1). This result is in sharp contrast to, for example, a substituted porphyrin in methanol where the relaxation is essentially complete in $<10 \mathrm{ps}$, but it does agree with the observation of 2DES for $\mathrm{Chl}$ a in methanol, where a long lived inhomogeneity was also reported. ${ }^{12}$

The residual inhomogeneity is largest for the viscous polar H-bonding EG, but very small for the fluid nonpolar cyclohexane. This component is of lower weight in the polar $\mathrm{H}$-bonding $\mathrm{MeOH}$ than in EG and reduces still further in the non-H-bonding polar solvents $A C$ and acetonitrile (ACN) (see Figure 5). These data suggest that there are specific features in EG which promotes inhomogeneous broadening. These could include its relatively high viscosity and diol character. We speculate that in EG there is slow (viscosity dependent) exchange between $\mathrm{H}$-bonding structures around the carbonyls associated with the $\pi$-delocalised ring, or associated with ligation of the central $\mathrm{Mg}^{+}$ion. However, in all solvents a degree of inhomogeneity exists in the ground state population that persists on the nanosecond time scale. On the basis of Figure 5, it appears that inhomogeneity is promoted by $\mathrm{H}$ bonding and, to a lesser extent, polarity.

All solvents contribute a high frequency $\mathrm{BO}$ to the spectral diffusion, peaking at or above $50 \mathrm{~cm}^{-1}$ in the spectral density (Figure 5). This can be traced to fast dynamics in the solvent. Such high frequency dynamics have been characterised in optical Kerr effect (OKE) studies for numerous pure liquids, including the specific solvents used here. ${ }^{26-29}$ The physical origin of this fast response is the non-diffusive librational motion of the molecule in a cage of its neighbours. ${ }^{30}$ The role of this contribution is further corroborated by the Gaussian relaxation measured at early times, which is typical of librational dynamics observed in OKE and photon echo studies. ${ }^{31-32}$ It has been shown in the past that non-diffusive librational motion contributes significantly to solvation dynamics measured from the rate of the Stokes shift in time-resolved fluorescence, and the FFCF recovered from three-pulse-echo peak-shift measurements. ${ }^{32-34}$ Thus, the appearance of this component in the 2DES spectral reshaping is expected and can, therefore, be reliably related to solvent dynamics. All of the polar solvents also exhibit additional relaxation on the picosecond and tens of picoseconds time scale. The pure liquids also exhibit such picosecond dynamics due to diffusive orientational motion, which is well characterised in both OKE measurements mentioned above and in observations of dielectric relaxation. ${ }^{35-36}$ However, there is no simple one-to-one correspondence 
between the $\mathrm{BO}$ damping times recovered here (Table $\mathrm{S} 1$ ) and the measured dielectric relaxation of the pure solvents. We suggest that the times recovered from the fits reflect the dynamics of the liquid in the presence of the multiple possible solvent-solute interactions presented by the amphiphilic Chl a solute.

\section{CONCLUSIONS}

The solvent dependence of the 2DES spectra for $\mathrm{Chl}$ a in a range of polar and nonpolar solvents have been investigated. For sub-picosecond population times oscillatory dynamics are observed. In some cases these were found across the 2D map and were assigned to solvent modes, which was confirmed by studies of different solvents and variation of solute concentration. However, molecular modes in $\mathrm{Chl}$ a were observed at positions on the 2D map predicted by Feynman diagrams at 255, 345 and $745 \mathrm{~cm}^{-1}$. The frequencies match previously reported $\mathrm{Chl}$ a vibrational frequencies. The power spectrum amplitude distribution of these vibrational modes as a function of excitationdetection frequencies was obtained from the rephasing 2D signal.

The dominant feature of the 2DES is the pure electronic transition, and the shape of that response was observed to evolve with time indicating spectral diffusion. The 2DES does not become homogeneous on a nanosecond time scale, indicating significant long lived inhomogeneous broadening in the ground state for Chla. The temporal evolution of the 2DES response was analysed in terms of a Brownian oscillator model and the spectral densities recovered. Importantly, the Stokes shift and linear absorption is also well described by this model. The spectral reshaping was quantified by a single parameter, namely the ellipticity, which was well reproduced by our calculations. The initial spectral reshaping is Gaussian like and correlates well with typical solvation response behaviour obtained by Kerr effect and photon-echo spectroscopies. The next fastest components of the response were assigned to solvent dynamics, modified by interactions with the solute. The extent of inhomogeneous broadening, the slowest response, was a strong function of solvent, being greater in $\mathrm{H}$-bonding and viscous media and small in nonpolar solvents. 


\section{Figure Legends}

Figure 1 (a) The 2DES spectrum measured for Chl a in methanol for a population time $T=100 \mathrm{fs}$. The right hand graph shows the absorption (red line) and fluorescence (green line) spectra of the sample as well as the laser spectrum (blue line) employed in this measurement. The dashed line indicates that the cross peak coincides with the $Q_{y}\left(S_{1}\left(v^{\prime}=0\right) \rightarrow S_{0}(v=1)\right)$ fluorescence emission peak. (b) Population time dependent evolution of 2D spectral amplitude for a pair of (excitation, detection) $=$ $(15770,14500)$ frequencies. (c) Power spectrum of curve shown in (b).

Figure 2. 2DES evolution for $\mathrm{Chl}$ a in acetone. The red numbers indicate the population time in picoseconds and the top panels show the absorption (red line) and the laser (blue line) spectra. Note the evolution of the 2D peak shapes from elongated to more circular, and the persistent lobe at the stimulated emission frequency (see text).

Figure 3. The ellipticity of the dominant central response as a function of population time for (a) cyclohexane (red line), (b) methanol (red line) and (c) ethylene glycol (red line) and, in each case (to provide a reference) compared with the response in acetone (blue line).

Figure 4. (a) Calculated 2DES data matching the results for $\mathrm{Chl}$ a in acetone. The top panels show the linear absorption spectrum (red lines) and the red numbers are the population times in ps. Good agreement is obtained for linear absorption spectrum, ellipticity and spectral Stokes shift, as shown in (b), where the black squares correspond to the experimental data and the red lines are the result from simulations.

Figure 5. Plot of the spectral densities, $C(\omega)$, underpinning the lineshape function analysis for the five different solvents studied in this work. The line assignments are as follows: acetone (blue), acetonitrile (red), methanol (green) ethylene glycol (magenta) and cyclohexane (orange). 
Figure 1

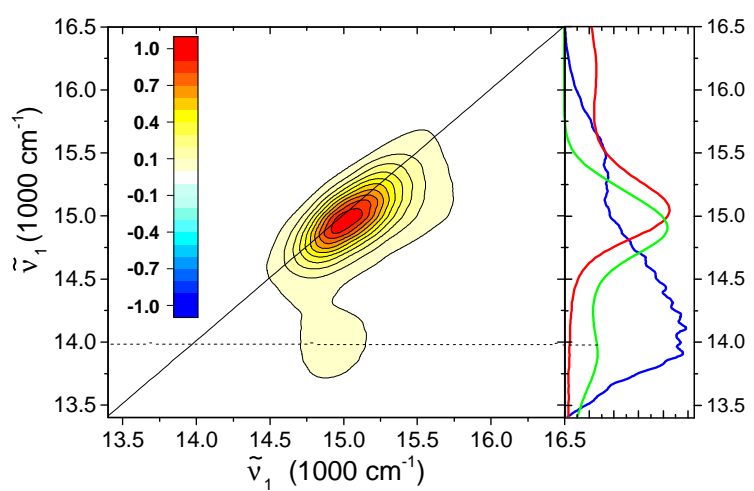

(a)

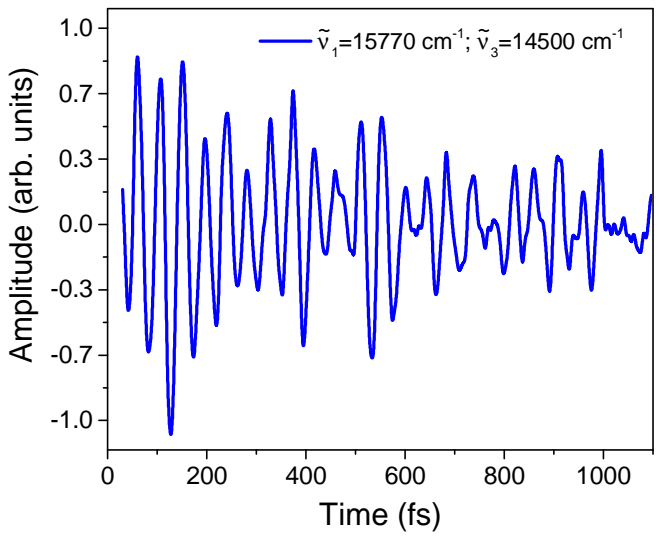

(b)

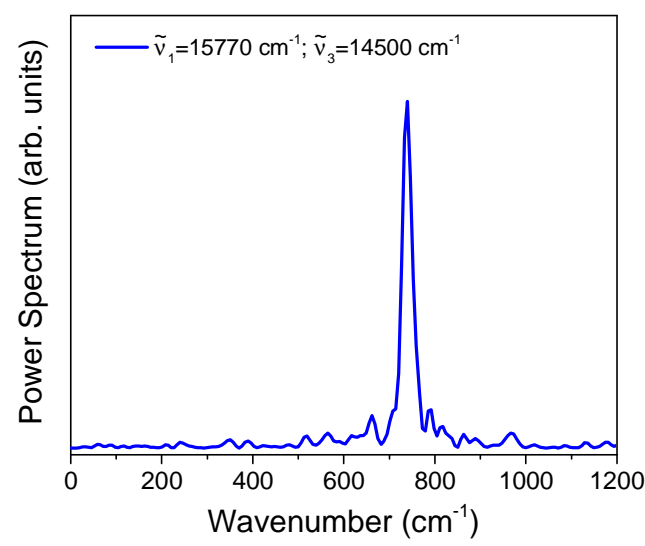

(c) 
Figure 2

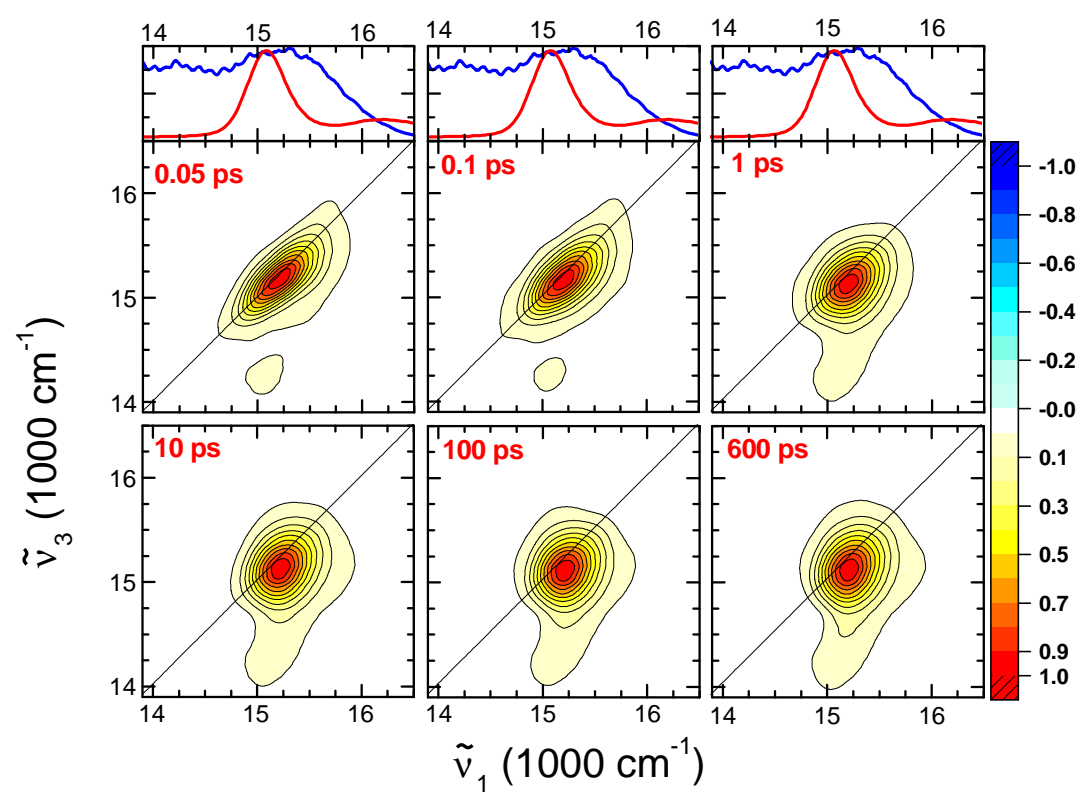


Figure 3

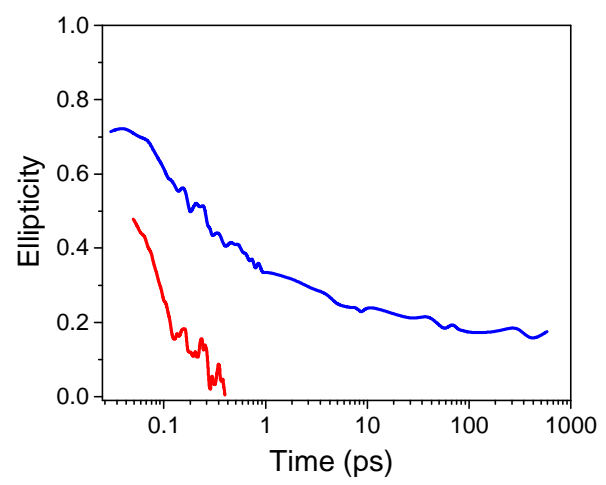

(a)

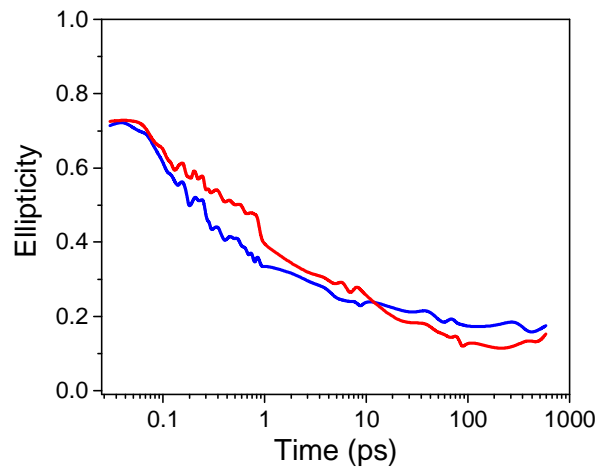

(b)

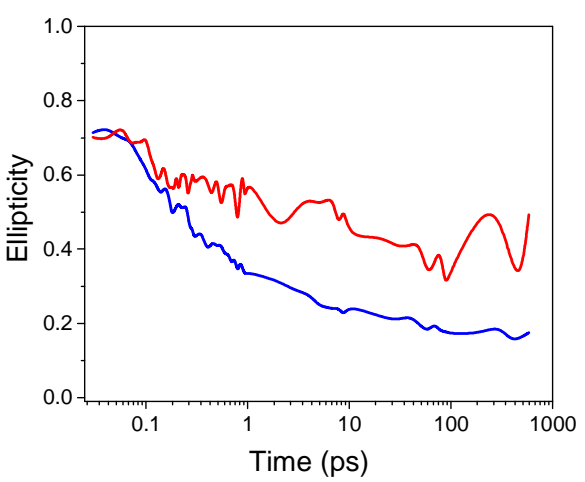

(c) 
Figure 4

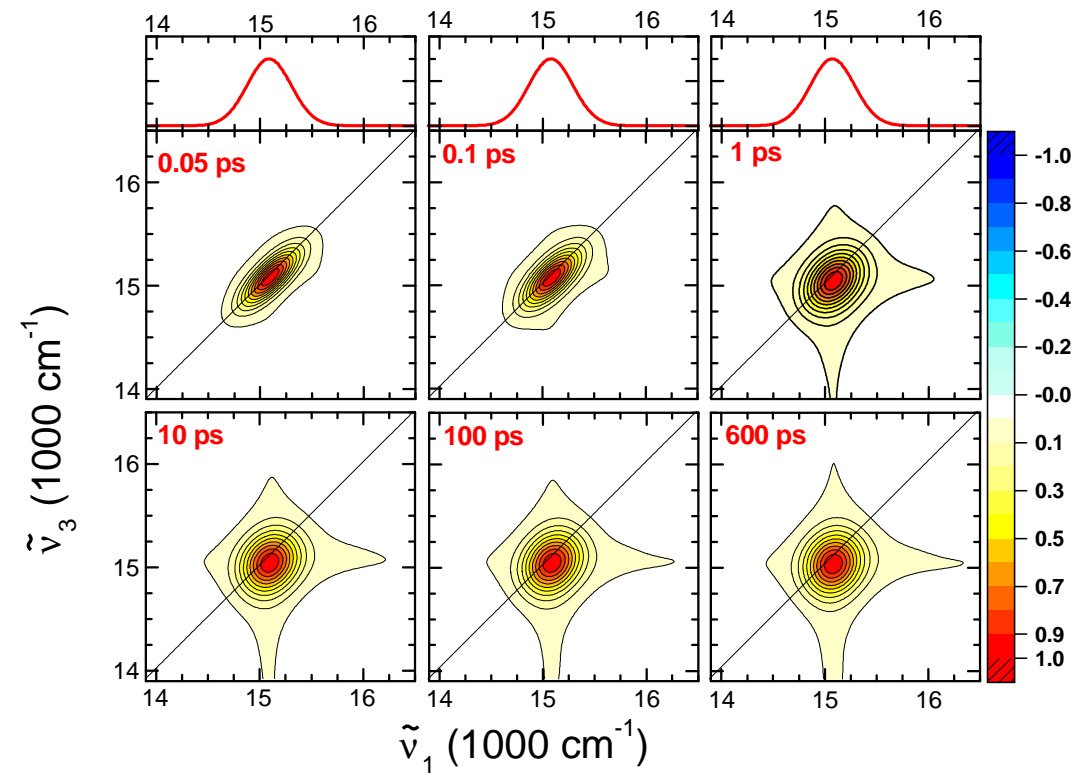

(a)
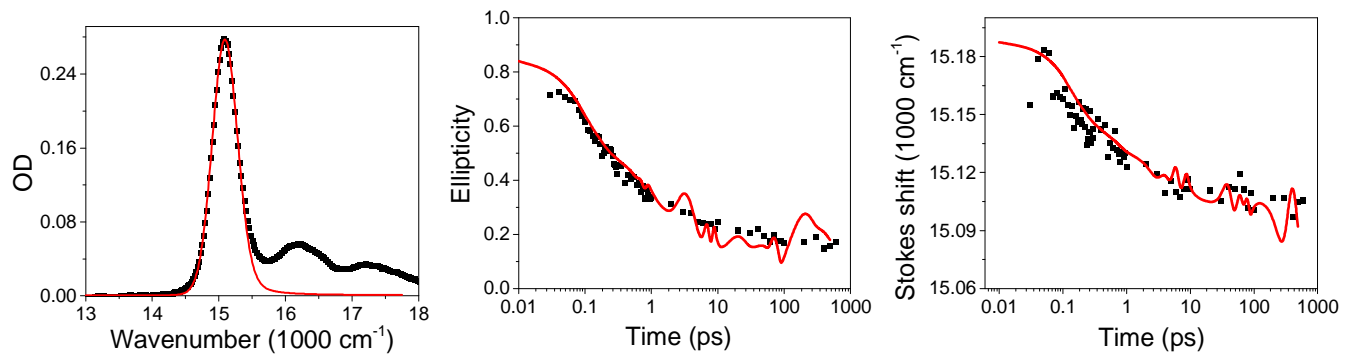

(b) 
Figure 5

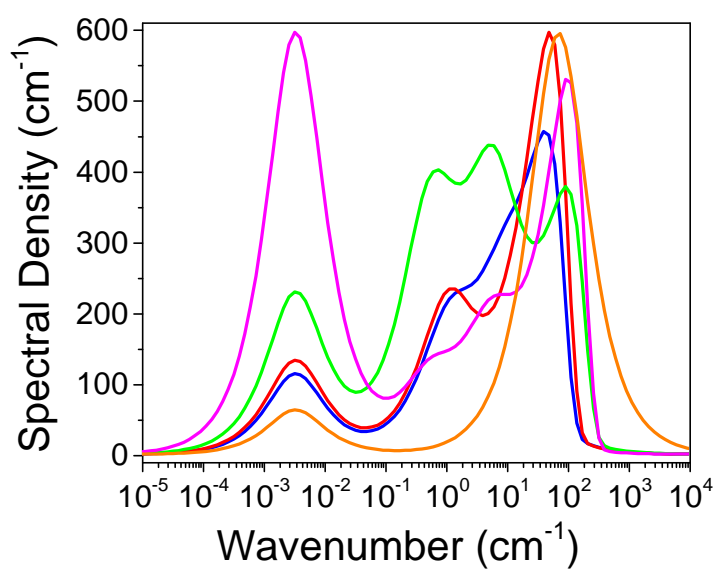




\section{References}

1. Fuller, F. D.; Pan, J.; Gelzinis, A.; Butkus, V.; Senlik, S. S.; Wilcox, D. E.; Yocum, C. F.; Valkunas, L.; Abramavicius, D.; Ogilvie, J. P. Vibronic coherence in oxygenic photosynthesis. Nature Chemistry 2014, 6 (8), 706-711.

2. Romero, E.; Augulis, R.; Novoderezhkin, V. I.; Ferretti, M.; Thieme, J.; Zigmantas, D.; van Grondelle, R. Quantum coherence in photosynthesis for efficient solar-energy conversion. Nat. Phys. 2014, 10 (9), 677-683.

3. Anna, J. M.; Ostroumov, E. E.; Maghlaoui, K.; Barber, J.; Scholes, G. D. Two-Dimensional Electronic Spectroscopy Reveals Ultrafast Downhill Energy Transfer in Photosystem I Trimers of the Cyanobacterium Thermosynechococcus elongatus. Journal of Physical Chemistry Letters 2012, 3 (24), 3677-3684.

4. Cheng, Y. C.; Fleming, G. R. Coherence quantum beats in two-dimensional electronic spectroscopy. J. Phys. Chem. A 2008, 112 (18), 4254-4260.

5. Read, E. L.; Schlau-Cohen, G. S.; Engel, G. S.; Wen, J. Z.; Blankenship, R. E.; Fleming, G. R. Visualization of excitonic structure in the Fenna-Matthews-Olson photosynthetic complex by polarization-dependent two-dimensional electronic spectroscopy. Biophys. J. 2008, 95 (2), 847-856.

6. Sengupta, S.; Wurthner, F. Chlorophyll J-Aggregates: From Bioinspired Dye Stacks to Nanotubes, Liquid Crystals, and Biosupramolecular Electronics. Accounts Chem. Res. 2013, 46 (11), 2498-2512.

7. Wurthner, F.; Kaiser, T. E.; Saha-Moller, C. R. J-Aggregates: From Serendipitous Discovery to Supramolecular Engineering of Functional Dye Materials. Angewandte Chemie-International Edition 2011, 50 (15), 3376-3410.

8. Renge, l.; Mauring, K. Spectral shift mechanisms of chlorophylls in liquids and proteins. Spectrochim Acta A 2013, 102, 301-313.

9. Martinsson, P.; Oksanen, J. A. I.; Hilgendorff, M.; Hynninen, P. H.; Sundstrom, V.; Akesson, E. Dynamics of ground and excited state chlorophyll a molecules in pyridine solution probed by femtosecond transient absorption spectroscopy. Chem. Phys. Lett. 1999, 309 (5-6), 386-394. 10. Hess, S.; Akesson, E.; Cogdell, R. J.; Pullerits, T.; Sundstrom, V. Energy transfer in spectrally inhomogeneous light-harvesting pigment-protein complexes of purple bacteria. Biophys. J. 1995, 69 (6), 2211-2225.

11. Du, J.; Teramoto, T.; Nakata, K.; Tokunaga, E.; Kobayashi, T. Real-Time Vibrational Dynamics in Chlorophyll a Studied with a Few-Cycle Pulse Laser. Biophys. J. 2011, 101 (4), 995-1003.

12. Wells, K. L.; Zhang, Z. Y.; Rouxel, J. R.; Tan, H. S. Measuring the Spectral Diffusion of Chlorophyll a Using Two-Dimensional Electronic Spectroscopy. J. Phys. Chem. B 2013, 117 (8), 22942299.

13. Heisler, I. A.; Moca, R.; Camargo, F. V. A.; Meech, S. R. Two-dimensional electronic spectroscopy based on conventional optics and fast dual chopper data acquisition. Rev. Sci. Instrum. 2014, 85 (6), 063103.

14. Vladkova, R. Chlorophyll a self-assembly in polar solvent-water mixtures. Photochem. Photobiol. 2000, 71 (1), 71-83.

15. Camargo, F. V. A.; Anderson, H. L.; Meech, S. R.; Heisler, I. A. Full characterization of vibrational coherence in a porphyrin chromophore by two-dimensional electronic spectroscopy. Journal of Physical Chemistry Letters 2014, Submitted.

16. Constantine, S.; Zhou, Y.; Morais, J.; Ziegler, L. D. Dispersed optical heterodyne detected birefringence and dichroism of transparent liquids. J. Phys. Chem. A 1997, 101 (30), 5456-5462.

17. Faeder, S. M. G.; Jonas, D. M. Two-dimensional electronic correlation and relaxation spectra: Theory and model calculations. J. Phys. Chem. A 1999, 103 (49), 10489-10505.

18. Schlau-Cohen, G. S.; Ishizaki, A.; Fleming, G. R. Two-dimensional electronic spectroscopy and photosynthesis: Fundamentals and applications to photosynthetic light-harvesting. Chem. Phys.

2011, $386(1-3), 1-22$. 
19. Roberts, S. T.; Loparo, J. J.; Tokmakoff, A. Characterization of spectral diffusion from twodimensional line shapes. J. Chem. Phys. 2006, 125 (8).

20. Milota, F.; Sperling, J.; Nemeth, A.; Mancal, T.; Kauffmann, H. F. Two-Dimensional Electronic Spectroscopy of Molecular Excitons. Accounts Chem. Res. 2009, 42 (9), 1364-1374.

21. Hamm, P.; Zanni, M. Concepts And Methods Of 2D Infrared Spectroscopy. Cambridge University Press: Cambridge, 2011.

22. Kwak, K.; Park, S.; Finkelstein, I. J.; Fayer, M. D. Frequency-frequency correlation functions and apodization in two-dimensional infrared vibrational echo spectroscopy: A new approach. J. Chem. Phys. 2007, 127 (12).

23. deBoeij, W. P.; Pshenichnikov, M. S.; Wiersma, D. A. System-bath correlation function probed by conventional and time-gated stimulated photon echo. J. Phys. Chem. 1996, 100 (29), 11806-11823.

24. Lazonder, K.; Pshenichnikov, M. S.; Wiersma, D. A. Easy interpretation of optical twodimensional correlation spectra. Opt. Lett. 2006, 31 (22), 3354-3356.

25. Khalil, M.; Demirdoven, N.; Tokmakoff, A. Coherent 2D IR spectroscopy: Molecular structure and dynamics in solution. J. Phys. Chem. A 2003, 107 (27), 5258-5279.

26. Shirota, H.; Fujisawa, T.; Fukazawa, H.; Nishikawa, K. Ultrafast Dynamics in Aprotic Molecular Liquids: A Femtosecond Raman-Induced Kerr Effect Spectroscopic Study. Bull. Chem. Soc. Jpn. 2009, 82 (11), 1347-1366.

27. Smith, N. A.; Meech, S. R. Femtosecond polarisability anisotropy relaxation and solvation dynamics - The cases of aniline and methanol. Faraday Discuss. 1997, 108, 35-50.

28. Shirota, H. Ultrafast dynamics of liquid poly(ethylene glycol)s and crown ethers studied by femtosecond Raman-induced Kerr effect spectroscopy. J. Phys. Chem. B 2005, 109 (15), 7053-7062.

29. Shirota, H.; Yoshihara, K.; Smith, N. A.; Lin, S. J.; Meech, S. R. Deuterium isotope effects on ultrafast polarisability anisotropy relaxation in methanol. Chem. Phys. Lett. 1997, 281 (1-3), 27-34.

30. Smith, N. A.; Meech, S. R. Optically heterodyne Detected Optical Kerr Effect (OHD-OKE): Application in Condensed phase dynamics. Int. Rev. Phys. Chem. 2002, 21 (1), 75-100.

31. Bagchi, B.; Jana, B. Solvation dynamics in dipolar liquids. Chem. Soc. Rev. 2010, 39 (6), 19361954.

32. Passino, S. A.; Nagasawa, Y.; Joo, T.; Fleming, G. R. Three-pulse echo peak shift studies of polar solvation dynamics. J. Phys. Chem. A 1997, 101 (4), 725-731.

33. Passino, S. A.; Nagasawa, Y.; Fleming, G. R. Three pulse stimulated photon echo experiments as a probe of polar solvation dynamics: Utility of harmonic bath models. J. Chem. Phys. 1997, 107 (16), 6094-6108.

34. Cho, M. H.; Scherer, N. F.; Fleming, G. R.; Mukamel, S. Photon-Echoes and Related 4-WaveMixing Spectroscopies Using Phase-Locked Pulses. J. Chem. Phys. 1992, 96 (8), 5618-5629.

35. Buchner, R.; Hefter, G. Interactions and dynamics in electrolyte solutions by dielectric spectroscopy. Phys. Chem. Chem. Phys. 2009, 11 (40), 8984-8999.

36. Park, S.; Joo, T. Diffractive optics based three-pulse photon echo peak shift studies of spectral diffusion in polar liquids: Evidence for long lived frequency correlations. J. Chem. Phys. 2009, 131 (16). 